\title{
The brain-heart connection: a multiple sclerosis relapse presenting as Takotsubo Syndrome. A case report and literature review
}

\author{
Andrea Dell'Aquila, Edoardo Sciatti, Enrico Vizzardi, Marco Metra \\ Cardiology Unit, University of Brescia and ASST Spedali Civili, Brescia, Italy
}

\begin{abstract}
We hereby report a puzzling case of multiple sclerosis (MS) relapse presenting as Takotsubo syndrome (TTS). Female, 42-years old, who presented herself to the Emergency Room of University Hospital "ASST Spedali Civili” of Brescia, Italy, for a severe headache and a non-ST-segment elevation acute coronary syndrome. Coronary angiogram showed no signs of coronary atherosclerosis. Upon further neurological evaluation, a diagnosis of MS relapse, related to TTS, was made, and treatment was started
\end{abstract}

Correspondence: Dr. Edoardo Sciatti, Cardiology Unit, University of Brescia and ASST Spedali Civili, Brescia, Italy. Tel. +39.030.3995536 - Fax: +39.030.3995013.

E-mail: edoardo.sciatti@gmail.com

Key words: Multiple sclerosis; Takotsubo; brain-heart connection.

Contributions: All the authors made a substantive intellectual contribution. All the authors have read and approved the final version of the manuscript and agreed to be accountable for all aspects of the work.

Conflict of interest: The authors declare that they have no competing interests, and all authors confirm accuracy.

Funding: there is no funding source.

Availability of data and materials: not available.

Ethics approval and consent to participate: No ethical committee approval was required for this case report by the Department, because this article does not contain any studies with human participants or animals. Informed consent was obtained from the patient included in this study.

Consent for publication: The patient gave her consent to use her personal data for the publication of this case report and any accompanying images.

Received for publication: 17 August 2019.

Accepted for publication: 5 March 2020.

${ }^{\circ}$ Copyright: the Author(s), 2020

Licensee PAGEPress, Italy

Monaldi Archives for Chest Disease 2020; 90:1153

doi: 10.4081/monaldi.2020.1153

This article is distributed under the terms of the Creative Commons Attribution Noncommercial License (by-nc 4.0) which permits any noncommercial use, distribution, and reproduction in any medium, provided the original author(s) and source are credited. accordingly. The patient was discharged after 12 days after the admission, free of symptoms, and without signs of neurological and cardiological active disease. A hallmark of TTS is its association with a preceding stressful event. It may also be connected to a wide variety of diseases, including neurological ones, such as stroke, intracranial bleeding, head trauma, migraine, and seizures. However, up to our knowledge, only few cases of MS-induced TTS were previously described. Whether it is plausible to consider TTS as an uncommon extra-neurological manifestation of MS is still debated, however all the evidence points in that direction, considering the central role of catecholamines in TTS pathogenesis. With this case report the authors hope to encourage research on this field and on the intricate topic of brain-heart connections.

\section{Introduction}

It is widely accepted that Takotsubo syndrome (TTS) may be triggered by emotional and physical stress [1]. In particular it may be associated with many conditions [2], including neurological diseases, such as stroke, intracranial bleeding [3], head trauma, migraine, and seizures [4]. We hereby report a puzzling case of central nervous system (CNS) demyelinating disease presenting as TTS.

\section{Case Report}

A 42-year-old female, smoker, with familiar history of multiple sclerosis (sister) and no history or family history of cardiovascular disease (CVD), presented herself to the Emergency Room (ER) of the University Hospital "ASST Spedali Civili" of Brescia, Italy, with severe headache (visual analog scale for pain=10/10), not responding to common analgesic medications. The patient reported an episode of left upper limb hypoesthesia lasting for almost a month, which happened a few months before. She also reported an episode, 2 years before, of binocular diplopia lasting a couple of days.

On arrival at the ER the pain subsided spontaneously, approximately two hours after its onset. A routine 12-lead electrocardiogram (ECG) showed sinus rhythm with ventricular bigeminism and infero-lateral (DII, DIII, aVF, aVL, V3-V6) ST segment depression of 0.3-0.5 $\mathrm{mV}$ (Figure 1).

On the blood tests leucocytosis (white blood cells $=19810 / \mathrm{uL}$ ) and a moderate elevation of myocardial necrosis markers (troponin $\mathrm{I}$, i.e. $\mathrm{TnI}=2.42 \mathrm{mcg} / \mathrm{L}$, creatine kinase-MB, i.e. $\mathrm{CK}-\mathrm{MB}=16$ $\mathrm{mcg} / \mathrm{L}$ ) were found. Myocardial necrosis markers presented a rise and fall pattern on subsequent testing at predefined time intervals, 
with peak $\mathrm{TnI}=7.39 \mathrm{mcg} / \mathrm{L}$ and peak $\mathrm{CK}-\mathrm{MB}=21 \mathrm{mcg} / \mathrm{L}$. Noncontrast brain computerized tomography scan ruled out intracranial bleeding. Chest X-ray showed no abnormalities.

The patient was admitted to the cardiac intensive care unit, and acetyl-salicylic acid and bisoprolol were administered, according to non-ST-segment elevation acute coronary syndromes guidelines ${ }^{5}$. Ticagrelor was also administered, considering the intention to perform a coronary angiogram (CAG). Transthoracic echocardiography (TTE) showed infero-lateral and inferior akinesia of the basal and mid segments of the left ventricular (LV) walls with preserved wall thickness, and hypokinesia of the midbasal infero-septal segments, with reduced LV ejection fraction ( $\mathrm{LVEF}=40 \%$ ). CAG showed no signs of coronary atherosclerosis (Figure 2). After the procedure the patient developed intense objective vertigo with nausea. Brain magnetic resonance imaging (MRI) was performed and showed multiple hyperintense FLAIR lesions, with no diffusion restriction, localised radially in the white matter adjacent to the lateral ventricles, in the right semioval centre, in the left juxtacortical frontal area and in the right paramedian bulbopontine sulcus. No abnormalities were found on the contrastenhanced brain MRI angiography (Figure 3). A complete spine MRI showed focal intramedullary T2 hyperintense lesions at C3 level and between $\mathrm{C} 4$ and $\mathrm{C} 5$ and a subtle hydrosyringomyelic cavity at D6 level.

Considering the findings suggestive of CNS demyelinating disease [6], the patient was transferred to the Neurology Inpatient Department. A complete neurological evaluation showed objective vertigo, binocular diplopia in the upward and rightward sustained gaze, grade 3 nystagmus in all gaze directions, with rightward and upward major amplitude jerks in sustained rightward gaze and leftward and upward major amplitude jerks in sustained leftward gaze, and subtle right eyelid ptosis. Immunosuppressant therapy with intravenous methylprednisolone was administered for 5 days, with subjective and objective benefit. Visually-evoked cortical potentials, brainstem acoustic-evoked potentials and somatosensory-evoked cortical potentials tests showed no abnormalities.

After cardiological evaluation anti-platelet medications were discontinued and beta-blocker therapy was up-titrated. Subsequent ECGs showed resolution of ST segment depression with inverted T waves, that normalized before discharge; QT interval remained stable on upper limits of normality (average QTc $=460 \mathrm{~ms}$ ). A subsequent TTE before discharge showed no abnormalities in left ventricular wall motion with normal LVEF. No new abnormalities were found in a complete brain and spine MRI scan at 6 days after the admission, and no contrast-enhancement was found in the lesions previously described.

The patient was discharged free of symptoms 12 days after admission with a diagnosis of TTS, according to the InterTAK Diagnostic Criteria [7,8], related to CNS demyelinating disease. Relapsing-Remitting Multiple Sclerosis was diagnosed according to the 2017 McDonald Criteria [9], being present both dissemination in space [6,9] in the MRI scans (periventricular and juxtacortical bulbopontine signal abnormalities), and dissemination in time $[9,10]$, considering that the anamnestic relapses (history of transient left upper limb hypoesthesia and transient binocular diplopia) were consistent with the non-enhancing lesions found in the MRI scans [6] .

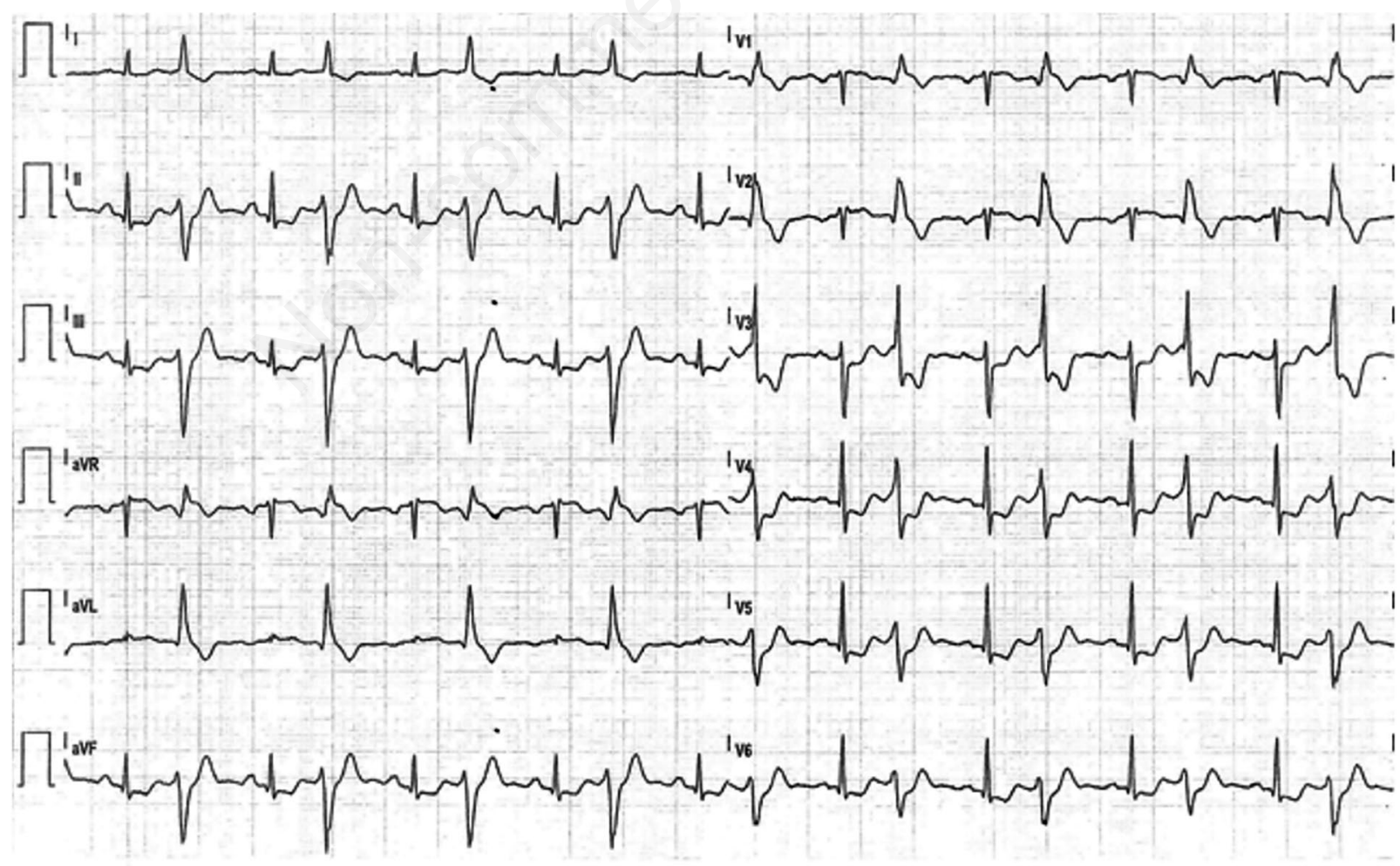

$25 \mathrm{~mm} / \mathrm{s} 10 \mathrm{~mm} / \mathrm{mV}$

Figure 1. Case report patient 12-lead electrocardiogram. Sinus rhythm with ventricular bigeminism and infero-lateral (DII, DIII, aVF, aVL, V3-V6) ST segment depression of 0.3-0.5 mV. 
On discharge, an appointment at 3 months was scheduled at the Outpatient Clinic of the local Multiple Sclerosis Neurology Centre, in order to re-assess the neurological status. A second appointment was scheduled in the same centre to perform lumbar puncture in order to complete the patient evaluation $[9,10]$

\section{Discussion}

Study estimates of prevalence and incidence of multiple sclerosis (MS) in European populations were highly heterogeneous. Heterogeneity could be found within regions or countries as well [9,11]. The most studied European countries were Italy and Great Britain. Prevalence in the latter ranged from 96 to more than 200 per 100,000 , while incidence rates ranged from 7.2 to 12.2 per 100,000 . In Italy, prevalence of MS is greater than 100 per 100,000, with incidence ranging from 3.4 to 6.8 per 100,000 [11]. MS is the third most common cause of disability in individuals 15 to 50 years of age, following only trauma and musculoskeletal disease. Considering that the calculation of disability-adjusted life years occurs mainly in the adult population of this age, MS could be considered not only a fairly common disease $[10,11]$, but also an important burden for the patient, family, health system, and society [11].

TTS is estimated to represent approximately $1-3 \%$ of all patients and $5-6 \%$ of female patients presenting with suspected acute coronary syndrome (ACS) [7,12]. It accounts for $0.02 \%$ of

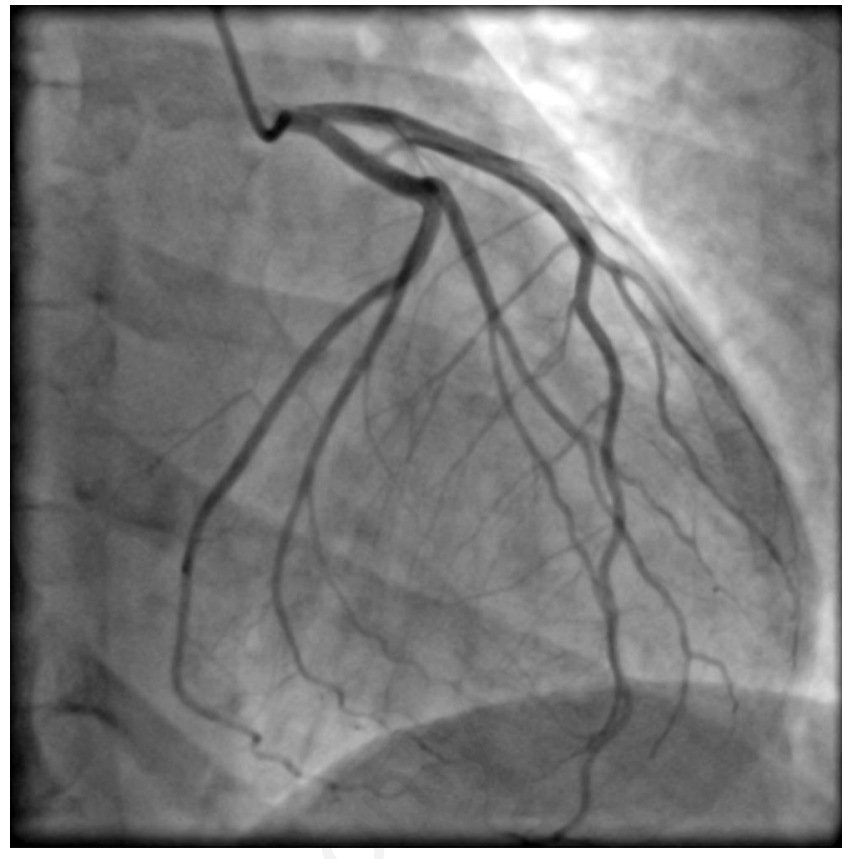

Figure 2. Case report patient coronary angiogram, selective cannulation of the left coronary artery. No signs of coronary artery atherosclerosis.
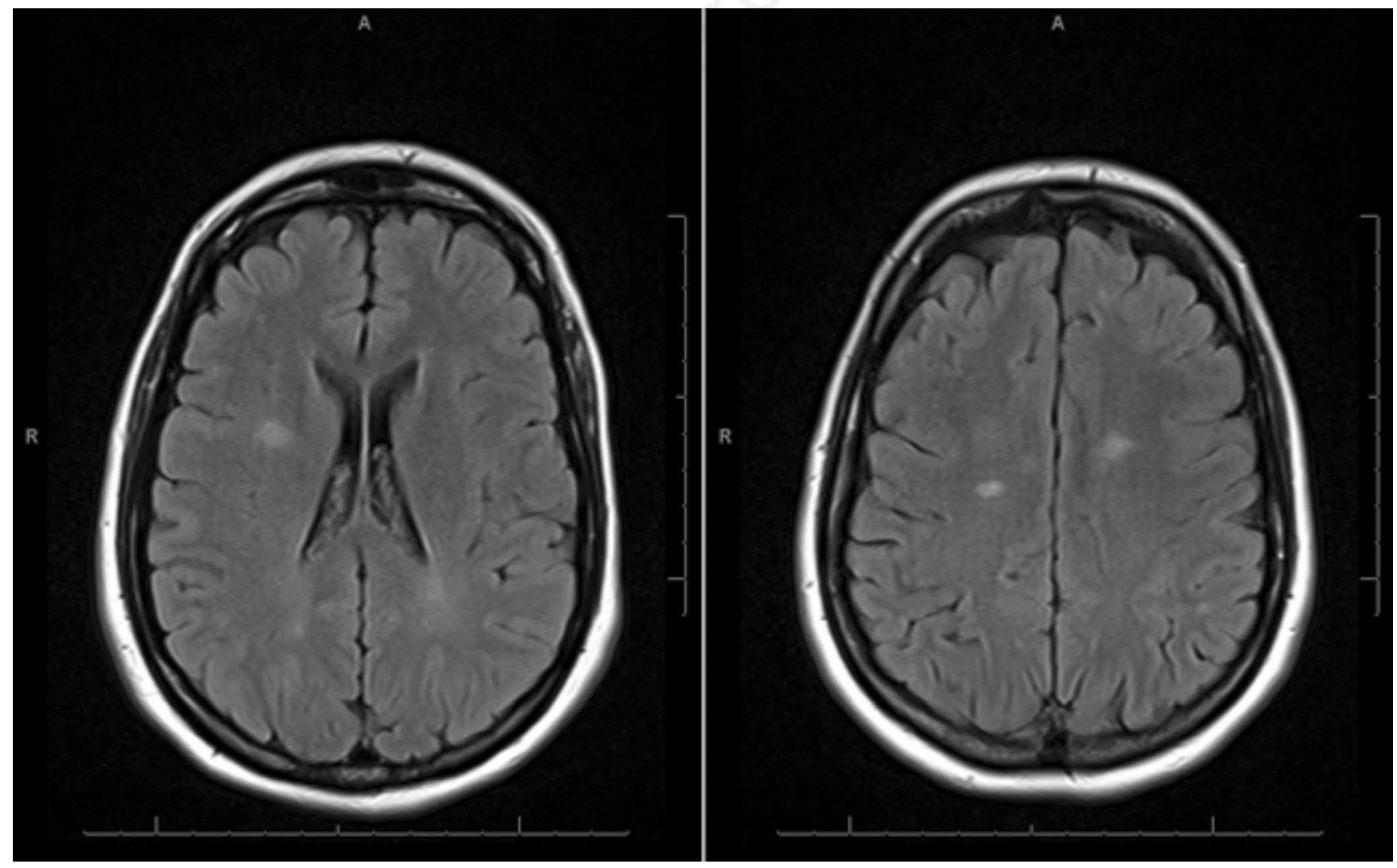

Figure 3. Case report patient brain magnetic resonance imaging (FLAIR sequences). Multiple hyperintense FLAIR lesions localised radially in the white matter adjacent to the lateral ventricles, in the right semioval centre and in the left juxtacortical frontal area. 
hospitalizations in the United States [7]. However TTS still remains an underappreciated and often misdiagnosed disorder up to this day $[7,8]$. Moreover, it is likely that subclinical TTS cases remain undetected, particularly in centres without coronary angiography on site [12]. TTS has a very low mortality, both in the short and mid term, but the risk of severe complications is not negligible $[7,13]$. Acute heart failure and cardiogenic shock, the most common ones $[1,13]$, may pose an important challenge for patients and clinicians attending to them. Arrhythmias and various cases of intracardiac thrombosis, with and without embolism, were described as well [7].

A hallmark of TTS is its association with a preceding stressful event. Initially, most reported triggers involved an emotional trauma [4]. As TTS became more widely appreciated, an association with physical stressors was also noted as well. In fact, physical triggers are more common than emotional stressors [7]. Furthermore triggers may be represented by a combination of emotional and physical stressors [2]. On the other hand, about one third of patients presents without evidence of an identifiable preceding stressful event $[2,4,7]$.

TTS triggers may be related to a great number of diseases, ranging from acute respiratory failure to pancreatitis [12]. Various medical procedures (e.g., electrophysiological testing, electrical cardioversion [14], dobutamine stress testing [15]) and treatments (e.g., chemotherapy, radiotherapy, sympathomimetic drugs, betaagonists for asthma or chronic obstructive lung disease) are known to be triggers [7]. Furthermore, to complicate the matter, ACS may also act as a trigger for TTS $[7,12]$. Neurological conditions, such as stroke, intracranial bleeding [3], head trauma, migraine, and seizures, also represent an important trigger for TTS [4,7]. Few cases of MS related to TTS were previously described as well [1619], however the link that this neurological condition has with transient myocardial dysfunction is still debated. To further complicate diagnosis, ECG abnormalities, including ventricular repolarization alterations, without $\mathrm{LV}$ dysfunction, were described in patients with various neurological conditions, such as ischaemic stroke, intracranial bleeding and brain tumours [20,21]. Moreover, the presence of ECG alterations, in particular T waves modifications and/or non-specific ST segment abnormalities, concomitant with subarachnoid haemorrhage was described to be predictive of LV dysfunction and pulmonary oedema [22].

The precise pathophysiology of TTS is still not completely understood [1], but excessive sympathetic stimulation plays without doubt a central role in its pathogenesis [23]. As already mentioned, emotional and/or physical stress acts in most cases as a trigger for TTS. This syndrome has been associated with catecholamine excess [12] and activated specific cerebral regions [24]. Clinical features of TTS and the various ballooning patterns can be caused by intravenous administration of catecholamines and beta-agonists $[15,23]$. Familial predisposition to this syndrome was described as well [25].

However, the exact mechanism by which catecholamine excess precipitates myocardial stunning is unknown. Several hypotheses have been proposed, the main ones being coronary vasospasm, both in epicardial [26] and small vessels [26,27], microcirculatory dysfunction [28,29], and catecholamine-induced toxicity on cardiomyocytes, i.e. metabolic injury [30]. Plasma levels of catecholamines and their metabolites were found to be two to three times higher in patients with transient LV apical ballooning syndrome compared to healthy controls [31]. Furthermore, it has been found that level of oxidative stress and plasma catecholamines changed proportionately with the extent of myocardial dysfunction, being twice as high in TTS patients compared to patients with acute myocardial infarction (AMI) [32]. Cases of pheochromocytoma presenting as TTS were described as well1, with one case of complete recovery of myocardial function after surgical removal of the tumour [33].

Catecholamine-mediated coronary vasospasm may play a role in TTS. Predisposition to coronary vasospasm using provocative testing, such as intracoronary acetylcholine, was demonstrated in approximately $30 \%$ of patients with a history of TTS [34]. Most studies where conducted in Japan, where vasospastic ischaemia is more common than in other countries, and the presence of spontaneous coronary vasospasm ranges from $1.8 \%$ to $27.7 \% 1$. The coronary vasospasm theory alone is insufficient to explain all cases of TTS, because the vast majority of patients do not show any evidence of epicardial vasospasm even when exposed to provocative agents [8].

In the few reported cases of MS presenting as TTS [16-19] the lesions causing the syndrome were all located in the medulla oblongata, where the cardiovascular centre is located. This centre, together with its sympathetic and parasympathetic connection to the cardiovascular system and the adrenal glands, provides modulation and fine tuning of heart rate, the strength of myocardial contractility, i.e. inotropism, and the dilatation and constriction of the peripheral vasculature, i.e. the vascular tone [35]. In the hereby-reported case, the patient suffered medulla oblongata damage as well, suggesting that cardiovascular centre lesions could trigger myocardial dysfunction by acute dysregulation of the cardiac sympathetic tone. Considering the clearly described connection between excessive sympathetic stimulation and myocardial stunning [23], this hypothesis may be plausible. Some authors go as far as to suggest that TTS could be an uncommon extra-neurological manifestation of MS [18], as a particular form of neurogenic pulmonary oedema (NPO) [22], called neuro-cardiac NPO [36]. While this link between the conditions and the exact physiopathology of TTS are still debated $[1,8,16]$, one could argue that this definition is not far from the truth. However further research is still needed in order to understand the exact mechanisms of myocardial stunning in TTS, and its possible link with neurological conditions, in particular with demyelinating disease.

Takotsubo syndrome was found to be associated with endothelial dysfunction, assessed by conducting ultrasound studies of the flow-mediated dilation (FMD) and the response to sublingual nitroglycerin of the brachial artery [1]. At presentation, patients with TTS have marked impairment in brachial artery FMD compared to those with infarction or healthy controls, which gradually improves over several weeks [37].

Endothelial dysfunction was found to be present after emotional stress and could be prevented by administering selective endothelinA receptor antagonists [38]. Furthermore other conditions linked to abnormal vasomotor function, such as migraine or Raynaud's phenomenon, were found to be associated to TTS [39]. Oxidative stress may also be a potential mechanism involved in myocardial dysfunction in TTS, considering that it may be linked to both endothelial dysfunction and catecholamine-induced cardiomyocyte toxicity. It was recently discovered that, in animal models, hydrogen sulphide attenuates cardiac dysfunction by alleviating oxidative stress [40].

In conclusion, the precise pathophysiology of TTS still remains a mystery, but it is clear that excessive sympathetic stimulation is central to its pathogenesis [1]. Many mechanisms may play a role in transient myocardial dysfunction observed in TTS8 and various stressors were found to be related to the onset of TTS [12]. However, up to our knowledge, only a few cases of MS presenting as TTS were previously described [16-19], and medulla oblongata lesions were described in each of these cases. Whether it is plausible 
to consider TTS as an uncommon extra-neurological manifestation of MS is still debated, however the presence of the cardiovascular centre in that specific region of the brain [35], together with the fact that its dysregulation could cause an excessive sympathetic stimulation, which in turn is central in TTS pathogenesis [1], corroborate this hypothesis. Furthermore it supports the already well-established connection between TTS and emotional or physical stressors, and in particular to neurological conditions, and the central role of catecholamines in TTS pathogenesis [8].

However, further research is still needed in order to better understand the pathophysiology of TTS, and to clarify its intimate link with neurological conditions, such as demyelinating disease.

\section{Conclusions}

MS is an underappreciated and often misdiagnosed disorder ACS $[8,10]$ and one of the main causes of disability in young, otherwise healthy individuals [11]. It has a very low mortality, but the risk of severe complications, mainly acute heart failure and cardiogenic shock, must not be neglected $[7,13]$.

The exact pathophysiology behind this syndrome is still unclear [1]. Up to our knowledge, only few cases of MS-induced TTS were described up to this day [16-19], and medulla oblongata lesions were described in each of these cases. Whether it is plausible to consider TTS as an uncommon extra-neurological manifestation of MS is still debated [16], however all the evidence gathered up to this day points in that direction, considering the central role of catecholamines in TTS pathogenesis [1].

With this case report the authors hope to encourage research on this field and on the intricate topic of brain-heart connections.

\section{References}

1. Vizzardi E, D'Aloia A, Zanini G, et al. Tako-tsubo-like left ventricular dysfunction: Transient left ventricular apical ballooning syndrome. Int J Clin Pract 2010;64:67-74.

2. Gianni M, Dentali F, Grandi AM, Set al. Apical ballooning syndrome or takotsubo cardiomyopathy: A systematic review. Eur Heart J 2006;27:1523-9.

3. Caretta G, Vizzardi E, Rovetta R, et al. The link between intracranial haemorrhage and cardiogenic shock: a case of Takotsubo cardiomyopathy. Acta Cardiol 2012;67:363-5.

4. Pilgrim TM, Wyss TR. Takotsubo cardiomyopathy or transient left ventricular apical ballooning syndrome: A systematic review. Int J Cardiol 2007;124:283-92.

5. Roffi M, Patrono C, Collet JP, et al. 2015 ESC Guidelines for the management of acute coronary syndromes in patients presenting without persistent st-segment elevation: Task force for the management of acute coronary syndromes in patients presenting without persistent ST-segment elevation of . Eur Heart J 2016;37:267-315.

6. Filippi M, Rocca MA, Arnold DL, et al. Use of imaging in multiple sclerosis. In: NE Gilhus, MP Barnes and M Brainin, editors. European Handbook of Neurological Management . New Castle: Blackwell Publishing Ltd.; 2011, pp. 35-51.

7. Ghadri JR, Wittstein IS, Prasad A, et al. International Expert Consensus Document on Takotsubo syndrome (Part I): Clinical characteristics, diagnostic criteria, and pathophysiology. Eur Heart J 2018;39:2032-46.
8. Ghadri JR, Wittstein IS, Prasad A, et al. International Expert Consensus Document on Takotsubo syndrome (Part II): Diagnostic Workup, outcome, and management. Eur Heart J. 2018;39:2047-62.

9. Aktas O, Wattjes MP, Stangel M, Hartung HP. Diagnosis of multiple sclerosis: revision of the McDonald criteria 2017. Lancet Neurol 2018;89:1344-54.

10. National Institute for Health and Clinical Excellence. Multiple sclerosis in adults: management. NICE Clin Guidel CG186 2014;:20-22.

11. Howard J, Trevick S, Younger DS. Epidemiology of multiple sclerosis. Neurol Clin 2016;34:919-39.

12. Ghadri JR, Ruschitzka F, Lüscher TF, Templin C. Takotsubo cardiomyopathy: still much more to learn. Heart 2014;100: 1804-12.

13. Vizzardi E, Bonadei I, Rovetta R, et al. Characteristics and midterm follow-up of a single-center population affected by Tako-Tsubo cardiomyopathy. J Cardiovasc Med2015;16:326-30.

14. Vizzardi E, Rovetta R, Bonadei I, et al. A case of Tako-Tsubo cardiomyopathy after electrical cardioversion. Minerva Med 2013;104:115-7.

15. D'Aloia A, Caretta G, Vizzardi E, et al. Heart failure syndrome due to dobutamine stress echocardiography: Tako-Tsubo induced-cardiomiopathy. Panminerva Med 2012;54 :53-5.

16. Prestipino E, Squitieri M, Razzolini L, Pastò L, Forleo P, Amato MP. A case of Takotsubo syndrome during a multiple sclerosis brainstem relapse. Mult Scler Relat Disord 2018;24:1-2.

17. Webb Tyler, Tania Reyna, Robert Blair RF. Multiple sclerosis exacerbation presenting as stress cardiomyopathy. Neurology2014;82(10 Supplement).

18. Midaglia L, Mariño JMJ, Sastre-Garriga J, et al. An uncommon first manifestation of multiple sclerosis: Tako-Tsubo cardiomyopathy. Mult Scler J 2016;22:842-6.

19. Bayer AD, Cahill JF, Rizvi SA. Multiple sclerosis relapse presenting as an acute cardiomyopathy. Mult Scler Relat Disord 2019;27:7-8.

20. Rudehill A, Olsson GL, Sundqvist K, Gordon E. ECG abnormalities in patients with subarachnoid haemorrhage and intracranial tumours. J Neurol Neurosurg Psychiatry 1987;50: 1375.

21. Koepp M, Kern A, Schmidt D. Electrocardiographic changes in patients with brain tumors. Arch Neurol1995;52:152-5.

22. Chen W-L, Huang C-H, Chen J-H, et al. ECG abnormalities predict neurogenic pulmonary edema in patients with subarachnoid hemorrhage. Am J Emerg Med 2016;34:79-82.

23. Abraham J, Mudd JO, Kapur N, et al. Stress cardiomyopathy after intravenous administration of catecholamines and betareceptor agonists. J Am Coll Cardiol 2009;53:1320-5.

24. Suzuki H, Matsumoto Y, Kaneta T, et al. Evidence for brain activation in patients with Takotsubo cardiomyopathy. Circ J 2014; 78:256-8.

25. Caretta G, Robba D, Vizzardi E, et al.Tako-tsubo cardiomyopathy in two sisters: a chance finding or familial predisposition? Clin Res Cardiol2015;104:614-6.

26. Fiol M, Carrillo A, Rodriguez A, Herrero J, García-Niebla J. Left ventricular ballooning syndrome due to vasospasm of the middle portion of the left anterior descending coronary artery. Cardiol 2012;19:314-6.

27. EA Martin, A Prasad, CS Rihal, et al. ndothelial function and vascular response to mental stress are impaired in patients with apical ballooning syndrome. J Am Coll Cardiol2010;56:1840-6.

28. Galiuto L, De Caterina AR, Porfidia A, et al. Reversible coronary microvascular dysfunction: A common pathogenetic 
mechanism in apical ballooning or Tako-Tsubo syndrome. Eur Heart J2010;31:1319-27.

29. Patel SM, Lerman A, Lennon RJ, Prasad A. Impaired coronary microvascular reactivity in women with apical ballooning syndrome (Takotsubo/stress cardiomyopathy). Eur Hear J Acute Cardiovasc Care 2013;2:147-52.

30. Wittstein IS. Stress cardiomyopathy: A syndrome of catecholamine-mediated myocardial stunning? Cell Mol Neurobiol2012;32:847-57.

31. Wittstein IS, Thiemann DR, Lima JA, et al. Neurohumoral features of myocardial stunning due to sudden emotional stress. N Engl J Med 2005;352:539-48.

32. Nanno T, Kobayashi S, Oda S, et al. A marked increase in myocardial oxidative stress associated with sympathetic hyperactivity is related to transient myocardial dysfunction in patients with Takotsubo cardiomyopathy. Circulation 2015;132:A14124

33. Sardesai SH, Mourant AJ, Sivathandon Y, et al. Phaeochromocytoma and catecholamine induced cardiomyopathy presenting as heart failure. Heart2007;63:234-7.

34. Tsuchihashi K, Ueshima K, Uchida T, et al. Transient left ventricular apical ballooning without coronary artery stenosis: A novel heart syndrome mimicking acute myocardial infarction. J Am Coll Cardiol2001;38:11-8.

35. Waldman SD. Pain Review. 1st ed. Philadelphia: Saunders; 2009; The medulla oblongata; p. 208.

36. Davison DL, Terek M, Chawla LS. Neurogenic pulmonary edema. Crit Care 2012;16:212.

37. Vasilieva E, Vorobyeva I, Lebedeva A, et al. Brachial artery flow-mediated dilation in patients with tako-tsubo cardiomyopathy. Am J Med 2011;124:1176-9.

38. Spieker LE, Hürlimann D, Ruschitzka F, et al. Mental stress induces prolonged endothelial dysfunction via endothelin-A receptors. Circulation2002;105:2817-20.

39. Scantlebury DC, Prasad A, Rabinstein A, Best PJM. Prevalence of migraine and Raynaud phenomenon in women with apical ballooning syndrome (Takotsubo or stress cardiomyopathy). Am J Cardiol2013;111:1284-8.

40. Zhang Z, Jin S, Teng X, et al. Hydrogen sulfide attenuates cardiac injury in takotsubo cardiomyopathy by alleviating oxidative stress. Nitric Oxide 2017;67:10-25. 